\title{
Vector Valued Baire Functions
}

\author{
H. R. Shatery and J. Zafarani
}

\begin{abstract}
In this paper, we study some properties of the Banach space $\beta_{\alpha}^{\circ}(X, E)$, consists of all Baire functions with relatively compact ranges from a perfectly normal space $X$ into a Banach space $E$. Moreover, we establish that if $\beta_{\alpha}^{\circ}(X, E)$ is linear isometric with $\beta_{\alpha}^{\circ}(Y, E)$, then some compactification of $X$ and $Y$ are homeomorphic.
\end{abstract}

Keywords: Baire functions, Borel functions, Banach-Stone theorem, isomorphisms MSC 2000: Primary 26A21, 28A05, secondary 46B04, 54C50, 54H05

\section{Introduction}

Let $K$ be a compact Hausdorff space and $E$ a Banach space. We designate by $C(K, E)$ (resp. $C(K)$ ), the space of all $E$-valued (resp. real-valued) continuous functions on $K$, provided with sup-norm. It is well known that $C(K) \otimes E$ is dense in $C(K, E)([8])$. The Banach-Stone Theorem says that for two locally compact Hausdorff spaces $X$ and $Y$, if $T: C_{\circ}(X) \rightarrow C_{\circ}(Y)$ is an isometric isomorphism, then there is a homeomorphism $\varphi: Y \rightarrow X$ and a continuous map $u: Y \rightarrow\{\lambda \in \mathbb{R}:|\lambda|=1\}$ such that $T(f)=u(f \circ \varphi)$, for every $f \in C_{\circ}(X)$. Here $C_{\circ}(X)$ denotes the set of all real continuous functions on $X$ which tends to zero at infinity $([2])$. Some other versions of Banach-Stone Theorem have been proved by many authors (cf. [2, 4, 5, 11]). The Banach-Stone Theorem also has been generalized for the vector valued continuous functions (cf. [2]). The aim of this article is to replace the class of continuous functions in the above results with the class of Baire functions with relatively compact ranges and to establish similar results.

For the remainder of this section, we introduce some definitions and basic facts. Throughout this paper, $X$ and $Y$ are two perfectly normal topological

H. R. Shatery: Department of Mathematics, University of Isfahan, Iran; shatery@math.ui.ac.ir

J. Zafarani: Department of Mathematics, University of Isfahan, Iran; jzaf@math.ui.ac.ir

ISSN 0232-2064 / \$2.50 (c) Heldermann Verlag Berlin 
spaces $[1,9,10]$. A topological space $X$ is perfectly normal if it is Hausdorff, and every closed subset is the zero set of some real continuous function.

For a finite ordinal number $\alpha$, we denote the Borel sets of multiplicative (additive) class $\alpha$ by $\mathcal{P}_{\alpha}\left(\mathcal{S}_{\alpha}\right)$, beginning with $\mathcal{P}_{0}=\mathcal{F}\left(\mathcal{S}_{0}=\mathcal{G}\right)$, as $([15])$ :

$$
\begin{aligned}
& \mathcal{P}_{\alpha}: \mathcal{F}, \mathcal{G}_{\delta}, \mathcal{F}_{\sigma \delta}, \ldots \\
& \mathcal{S}_{\alpha}: \mathcal{G}, \mathcal{F}_{\sigma}, \mathcal{G}_{\delta \sigma}, \ldots
\end{aligned}
$$

As $X$ is perfectly normal, so the $\mathcal{P}_{\alpha}$ 's $\left(\mathcal{S}_{\alpha}\right.$ 's) form a chain and $\mathcal{F} \subseteq \mathcal{G}_{\delta}$, similar to the metric case (see [15]). For each $A \in \mathcal{P}_{\alpha}$, there exists a sequence $\left(G_{n}\right)_{n=1}^{\infty} \subseteq$ $\mathcal{S}_{\alpha-1}$ such that $A=\cap_{n=1}^{\infty} G_{n}$. For additive sets, " $\mathcal{S}$ ", " $\mathcal{P}$ ", and " $\cap$ " are replaced by "P ", " $\mathcal{S}$ ", and " $\cup$ ", respectively (see $[15, \S 30]$ for details). The ambiguous set of class $\alpha$ is denoted by $\mathcal{H}_{\alpha}$ [15] and defined as $\mathcal{H}_{\alpha}=\mathcal{S}_{\alpha} \cap \mathcal{P}_{\alpha}$. One can easily see that the separation theorem for Borel sets in metric spaces is valid for Borel sets in perfectly normal topological spaces $([15, \S 30])$.

Let $X$ be a topological space and $\beta_{0}(X)=C(X)$ be the set of all real valued continuous functions on $X$. Then for each ordinal $\alpha$, we define Baire functions of class $\alpha$ as

$$
\beta_{\alpha}(X)=\left\{f: X \rightarrow \mathbb{R}: \begin{array}{l}
\text { there exists }\left(f_{n}\right)_{n=1}^{\infty} \subseteq \beta_{\alpha-1}(X) \text { such } \\
\text { that } \lim f_{n}(x)=f(x) \text { for each } x \in X
\end{array}\right\} .
$$

We also define Borel functions of class $\alpha$ as

$$
B_{\alpha}(X)=\left\{f: X \rightarrow \mathbb{R}: f^{-1}(F) \in \mathcal{P}_{\alpha} \text { for each closed set } F \text { in } \mathbb{R},\right\}
$$

When $X$ is a perfectly normal space, then by the same induction as in [17], $\beta_{\alpha}(X) \subseteq B_{\alpha}(X)$. It's obvious that $B_{\alpha}(X) \subseteq B_{\alpha+1}(X)$.

More generally, for a Banach space $E$, suppose that $C^{\circ}(X, E)$ is the set of all of $E$-valued continuous functions with relatively compact ranges. Now, we define

$$
\begin{aligned}
& \beta_{0}^{\circ}(X, E)=C^{\circ}(X, E) \\
& \beta_{\alpha}^{\circ}(X, E)=\left\{f: X \rightarrow E: \begin{array}{l}
f \text { is the point-wise limit of some sequence in } \\
\beta_{\alpha-1}(X, E), \text { the range of } f \text { is relatively compact }
\end{array}\right\} \\
& B_{\alpha}^{\circ}(X, E)=\left\{f: X \rightarrow E: \begin{array}{l}
f^{-1}(F) \in \mathcal{P}_{\alpha} \text { for each } F, \text { closed in } E \\
\text { and the range of } f \text { is relatively compact }
\end{array}\right\}
\end{aligned}
$$

equipped with the sup-norm.

Here, we give a Baire- $\alpha$ characterization of $\mathcal{H}_{\alpha}$ elements in $X$. Let $H$ be a subset of $X$. We denote the characteristic function of $H \subseteq X$ by $\chi_{H}$.

Lemma 1.1. Let $X$ be a perfectly normal space and $E$ a Banach space with $0 \neq e \in E$. Then $H \in \mathcal{H}_{\alpha}$ if and only if $e \chi_{H} \in \beta_{\alpha}(X, E)$. 
Proof. We prove by induction. Suppose $H$ is in $\mathcal{H}_{\alpha}$. As $X$ is perfectly normal, then it is normal and the theorem is true for $\alpha=1$. Suppose that the statement holds for $(\alpha-1)$. There is a nondecreasing sequence $\left(F_{n}\right)_{n=1}^{\infty}$ of elements $\mathcal{P}_{\alpha-1}$ in $X$ and a nonincreasing sequence $\left(G_{n}\right)_{n=1}^{\infty}$ of elements $\mathcal{S}_{\alpha-1}$ in $X$ such that $\cup_{n=1}^{\infty} F_{n}=H=\cap_{n=1}^{\infty} G_{n}$. For each positive integer $n, F_{n} \subseteq G_{n}$. Thus there exists a separating set $H_{n} \in \mathcal{H}_{\alpha-1}$ such that $F_{n} \subseteq H_{n} \subseteq G_{n}([15], \S 30)$. By our induction hypothesis, the function $f_{n}=e \chi_{H_{n}} \in \beta_{\alpha-1}(X, E)$. This function trivially satisfies $f_{n}\left(F_{n}\right)=\{e\}$ and $f_{n}\left(G_{n}^{c}\right)=\{0\}$. Hence, $e \chi_{H}$ is the point-wise limit of $f_{n}$. The proof of the other direction is obvious and is omitted.

Definition 1.2. Let $X$ be a perfectly normal topological space and $E$ a Banach space, we define

$$
\Sigma_{\alpha, E}(X)=\left\{\sum_{i=1}^{n} e_{i} \chi_{H_{i}}: n \in \mathbb{N}, e_{i} \in E \text { and } H_{i} \in \mathcal{H}_{\alpha} \text { in } X \text { for each } i\right\},
$$

and when $E$ equals the real numbers, we denote it simply by $\Sigma_{\alpha}(X)$.

In the following theorem, we give an approximation theorem for Baire functions by simple functions.

Theorem 1.3. Let $X$ be a perfectly normal topological space. For a Frechet space $E$, the uniform closure of $\Sigma_{\alpha, E}(X)$ is $\beta_{\alpha}^{\circ}(X, E)$.

Proof. Suppose that $E$ is a Banach space and $f \in \beta_{\alpha}^{\circ}(X, E)$. As range $(f)$ is relatively compact, therefore there exists a countable set $Z \subseteq E$ such that range $(f)$ is in the norm closure of $Z$. For each positive integer $n$, let $\mathcal{C}_{n}$ be the collection of open balls of radius $\frac{1}{n}$ in $E$ with members of $Z$ as their centers. Hence range $(f)$ is covered by finite members of $\mathcal{C}_{n}$, denoted by $\mathcal{B}_{n}$, labeled by the finite set $I_{n}$. Set $\mathcal{B}_{n}^{\prime}=f^{-1}\left(\mathcal{B}_{n}\right)$. Thus $\mathcal{B}_{n}^{\prime}$ is a covering of $X$ and each of its elements belongs to $\mathcal{S}_{\alpha}$. Hence, its members are $\mathcal{P}_{\alpha}$ and $X$ has a finite refinement consisting of mutually disjoint elements of $\mathcal{H}_{\alpha}$ sets. Therefore, $\mathcal{A}_{n}=\left\{A_{i, n}: i \in I_{n}\right\}$ is a refinement of $\mathcal{B}_{n}^{\prime}$ with $\mathcal{H}_{\alpha}$ sets. We can suppose that for any $n \geq 2, \mathcal{A}_{n}$ refines $\mathcal{A}_{n-1}$.

Now, for each $n \geq 1$ and each $i \in I_{n}$, choose $y_{i, n} \in f\left(A_{i, n}\right)$. Let $x \in X$ and for each $n \geq 1$ let $i(x, n) \in I_{n}$ be such that $x \in A_{i(x, n), n}$. If $m \geq n$, then $A_{i(x, m), m} \subseteq A_{i(x, n), n}$. Consequently, since $f\left(A_{i(x, n), n}\right)$ has a diameter at most $\frac{2}{n}$, $\left\{y_{i(x, n), n}: n \geq 1\right\}$ is a Cauchy sequence. Now, for each $n \in \mathbb{N}$, we define $f_{n}=\sum_{i \in I_{n}} y_{i, n} \chi_{A_{i, n}}$. It is obvious that the $f_{n}$ 's are in $\Sigma_{\alpha, E}(X)$, and $f$ is the uniform limit of the $f_{n}$ 's.

For the case when $E$ is a Frechet space, it is enough to work with a countable collection of semi-norms that introduce its topology.

By the above theorem, it is obvious that $\mathcal{S}_{\alpha}=\mathcal{P}_{\alpha}$ if and only if $\beta_{\alpha}^{\circ}(X, E)=$ $\beta_{\alpha+1}^{\circ}(X, E)$. 


\section{A Banach-Stone type theorem for Baire functions}

In this section, we will establish a Banach-Stone type theorem for Baire classes. For this aim, we define $\alpha$-continuous and $\alpha$-homeomorphism maps between topological spaces. For $f: X \rightarrow Y$, the following three statements are equivalent (see, e.g., [13]):

(1) The inverse image by $f$ of every $\mathcal{P}_{\alpha}$ set in $Y$ is a $\mathcal{P}_{\alpha}$ set in $X$.

(2) The inverse image by $f$ of every $\mathcal{S}_{\alpha}$ set in $Y$ is an $\mathcal{S}_{\alpha}$ set in $X$.

(3) The inverse image by $f$ of every $\mathcal{H}_{\alpha}$ set in $Y$ is an $\mathcal{H}_{\alpha}$ set in $X$.

If one of the three above equivalent statements holds, then we say that $f$ is an $\alpha$-continuous map. Let $f$ be bijective. If $f$ and $f^{-1}$ are both $\alpha$-continuous, then we say that $f$ is an $\alpha$-homeomorphism between $X$ and $Y$, and we say that $X$ and $Y$ are $\alpha$-homeomorphic. Now, we mention some results for these classes of functions (see [13]). It is trivial that every continuous function is $\alpha$-continuous. Let $X, Y$ and $Z$ be three topological spaces. Suppose that $\alpha$ and $\beta$ are two finite ordinal numbers such that $\alpha \leq \beta$. Then:

(1) If $f: X \rightarrow Y$ and $g: Y \rightarrow Z$ are $\alpha$-continuous and $\beta$-continuous, respectively, then $g \circ f: X \rightarrow Z$ is $\beta$-continuous.

(2) If $f \in B_{\alpha}(X)$ and $\tau: Y \rightarrow X$ is $\alpha$-continuous, then $f \circ \tau \in B_{\alpha}(Y)$. Also if $\tau: X \rightarrow Y$ is an $\alpha$-homeomorphism, then $\theta: B_{\alpha}^{\circ}(Y) \rightarrow B_{\alpha}^{\circ}(X)$ defined by $\theta(f)=f \circ \tau$ is a Banach algebra isometry.

It is obvious that every homeomorphism is an $\alpha$-homeomorphism for each $\alpha \geq 1$. Now, we are ready to prove a Banach-Stone type theorem for Borel classes and therefore, for Baire classes of finite order (cf. [18, 19]). In [7, 12, 13], it has been proved that for completely regular spaces $X$ and $Y$, if the Banach spaces $\beta_{\alpha}^{\circ}(X)$ and $\beta_{\alpha}^{\circ}(Y)$ are isometric, then some compactifications of $X$ and $Y$ are homeomorphic. In the following theorem, by a direct proof, we give a relation between these two spaces and not between their compactifications when $X$ and $Y$ are two perfectly normal spaces.

Theorem 2.1. Let $X$ and $Y$ be two perfectly normal spaces. If $\varphi: B_{\alpha}^{\circ}(Y) \rightarrow$ $B_{\alpha}^{\circ}(X)$ is a surjective isometric ring isomorphism, then there exists an $\alpha$ homeomorphism $\tau: X \rightarrow Y$ such that for each $f$ in $B_{\alpha}^{\circ}(Y)$, we have $\varphi(f)=f \circ \tau$.

Proof. Since $\varphi$ is a ring homomorphism, for each $H \in \mathcal{H}_{\alpha}$ in $Y, \chi_{H} \chi_{H}=\chi_{H}$ and we have $\varphi\left(\chi_{H}\right) \varphi\left(\chi_{H}\right)=\varphi\left(\chi_{H}\right)$. Consequently, the value of $\varphi\left(\chi_{H}\right)$ are 0 or 1 . Hence, it must be a characteristic function of exactly one member of $\mathcal{H}_{\alpha}$ in $X$. We denote this member by $\psi(H)$. So we deduce that

$$
\psi: H_{\alpha} \text { in } Y \rightarrow H_{\alpha} \text { in } X \text { and } \varphi\left(\chi_{H}\right)=\chi_{\psi(H)} .
$$

We prove that $\psi$ is a surjective Boolean isomorphism. First, we prove $\psi$ is surjective. Let $K$ be $\mathcal{H}_{\alpha}$ in $X$. As $\varphi$ is surjective, there exists an $f$ in $B_{\alpha}^{\circ}(Y)$ 
such that $\varphi(f)=\chi_{K}$, and $\varphi(f) \phi(f)=\chi_{K} \chi_{K}=\chi_{K}=\varphi(f)$. The function $\varphi$ is one to one and $\varphi\left(f^{2}\right)=\varphi(f)$, therefore $f^{2}=f$. Consequently, $f$ is the characteristic function of an $H \in \mathcal{H}_{\alpha}$ in $Y$. Also, $\psi$ is one to one. Let $H_{1}$ and $H_{2}$ be two $\mathcal{H}_{\alpha}$ elements in $Y$. We have $\chi_{\psi\left(H_{1}\right)} \chi_{\psi_{\left(H_{2}\right)}}=\varphi\left(\chi_{H_{1}}\right) \varphi\left(\chi_{H_{2}}\right)=$ $\varphi\left(\chi_{H_{1}} \chi_{H_{2}}\right)=\varphi\left(\chi_{H_{1} \cap H_{2}}\right)$. Consequently, $\chi_{\psi\left(H_{1}\right) \cap \psi\left(H_{2}\right)}=\chi_{\psi\left(H_{1} \cap H_{2}\right)}$, therefore, $\psi\left(H_{1} \cap H_{2}\right)=\psi\left(H_{1}\right) \cap \psi\left(H_{2}\right)$.

In the other way, $\chi_{H_{1}}+\chi_{H_{2}}-\chi_{H_{1} \cap H_{2}}=\chi_{H_{1} \cup H_{2}}$. Similar to the previous case, this implies that $\psi\left(H_{1} \cup H_{2}\right)=\psi\left(H_{1}\right) \cup \psi\left(H_{2}\right)$. From the above argument, it is obvious that $\psi$ is one to one and onto (if $\psi(H)=\emptyset=\psi(\emptyset)$, then $\varphi\left(\chi_{H}\right)=$ $\left.\varphi\left(\chi_{\emptyset}\right)\right)$. As the spaces $X$ and $Y$ are perfectly normal spaces and $\alpha \geq 1$, therefore, all finite subsets of $X$ and $Y$ belong to $\mathcal{H}_{\alpha}$. If $H_{1} \subseteq H_{2}$, then it is obvious that $\psi\left(H_{1}\right) \subseteq \psi\left(H_{2}\right)$. Now, we claim that $\psi(\{y\})$ is a subset of $X$ with exactly one element for each $y$ in $Y$. Suppose $\psi(\{y\})=A$ and $A$ has at least two distinct elements. Let $a$ be in $A$. So there exists a set $F \varsubsetneqq\{y\}$ such that $\psi(F)=A-\{a\} \neq \emptyset$, because $\psi$ preserves order. Consequently, $F$ must be $\emptyset$, which contradicts the fact that $\psi$ is a one to one map. Let $\tau: X \rightarrow Y$ be defined by $\psi^{-1}$, then $\tau$ is a function that assigns a two sided correspondence between the $\mathcal{H}_{\alpha}$ members of $X$ and $Y$ and therefore, it is an $\alpha$-homeomorphism.

But $\varphi$ is a ring isomorphism, so for each rational number $a=\frac{p}{q}, \varphi\left(a \chi_{H}\right)=$ $a \varphi\left(\chi_{H}\right)$ and $\varphi$ is an isometry, thus it is continuous and therefore, for each real number $r$, we have $\varphi\left(r \chi_{H}\right)=r \chi_{\psi(H)}=r \chi_{\tau^{-1}(H)}$ for $H \in \mathcal{H}_{\alpha}$ in $Y$.

In the other side, $\psi$ is a Boolean isomorphism so the following relation holds:

$$
\varphi\left(\sum_{i=1}^{m} a_{i} \chi_{H_{i}}\right)=\sum_{i=1}^{m} a_{i} \chi_{\psi\left(H_{i}\right)} .
$$

By (1), we have $\varphi(g)=g \circ \tau$ for each $g \in \Sigma_{\alpha}(Y)$. Theorem 1.3 implies that the uniform closure of $\Sigma_{\alpha}(Y)$ is $B_{\alpha}^{\circ}(Y)$. Hence if $f$ is in $B_{\alpha}^{\circ}(Y)$, then there exists a sequence $\left(f_{n}\right)_{n=1}^{\infty}$ in $\Sigma_{\alpha}(Y)$ such that unif $-\operatorname{Lim} f_{n}=f$. Consequently, unif $-\operatorname{Lim} \varphi\left(f_{n}\right)=\varphi(f)$. So we have $\varphi(f)=$ unif $-\operatorname{Lim} f_{n} \circ \tau=f \circ \tau$.

\section{Isomorphisms between vector valued Baire functions}

Choban [6] investigated some of the properties of the following compactification for a completely regular space $X$ denoted by $b_{\alpha} X$. Let $P X$ be the set $X$ with the topology generated by the $\mathcal{G}_{\delta}$ sets in $X$ for a completely regular space $X$. The topology of the space $P X$ is called the Baire topology of the space $X$. If $\beta_{1}(X) \subseteq K \subseteq \beta_{\Omega}(X)$, where $\Omega$ is the first uncountable ordinal number, then $P X=\left(X, \tau_{K}\right)$, the weakest topology on $X$ generated by $K$. We define by $b_{\alpha} X$ the compactification of $\left(X, \tau_{\beta_{\alpha}(X)}\right)$. The compact space $b_{\alpha} X$ is called the maximal ideal space of the $\alpha$-th Baire class $\beta_{\alpha}(X)$. 
We are going to give a homeomorphic relation between $X$ and $Y$ when the vector valued Baire functions on $X$ and $Y$ are linear isometric. Suppose that $X$ is a perfectly normal topological space and $E$ is a real Banach space. In this part, we use the notation of ([8, Chapter I])to obtain the dual of $\beta_{\alpha}^{\circ}(X)$. Let $F$ : $\mathcal{H}_{\alpha} \rightarrow E$ be a bounded finitely additive vector measure on the field $\mathcal{H}_{\alpha}$ subsets of $X$. We denote by $V M\left(\mathcal{H}_{\alpha}, E\right)$ the space of all bounded finitely additive vector measures from $\mathcal{H}_{\alpha}$ into Banach space $E$ equipped with semivariation norm. Every operator $T$ in $\mathrm{E}\left(\beta_{\alpha}^{\circ}(X), E\right)$ is related to an element $F$ in $V M\left(\mathcal{H}_{\alpha}, E\right)$ with the correspondence

$$
T_{F}(f)=\int_{X} f d F
$$

for every $f$ in $\beta_{\alpha}^{\circ}(X, E)$.

The following theorem is exactly like that of vector valued continuous functions defined on a compact space [8].

Theorem 3.1. For a perfectly normal space $X$ and a Banach space $E$, we have $\beta_{\alpha}^{\circ}(X, E)=\beta_{\alpha}^{\circ}(X) \check{\otimes} E$.

Proof. We use the notation of [8]. We define $\theta: \beta_{\alpha}^{\circ}(X) \otimes E \rightarrow \beta_{\alpha}^{\circ}(X, E)$ as

$$
\theta\left(\sum_{i=1}^{n} f_{i} \otimes e_{i}\right)(x)=\sum_{i=1}^{n} e_{i} f_{i}(x) .
$$

The map $\theta$ is well-defined because the $f_{i}$ 's are bounded, and functions in the range of $\theta$ have finite dimension ranges. One can show easily that this linear map is an isometry, and the range of $\theta$ contains all functions in $\Sigma_{\alpha, E}(X)$. But by Theorem 1.3, $\Sigma_{\alpha, E}(X)$ is dense in $\beta_{\alpha}^{\circ}(X, E)$ and therefore, $\theta$ is an onto isometry.

By the above theorem, the functional $\psi$ is in the dual of $\beta_{\alpha}^{\circ}(X, E)$ if and only if there exists regular Borel measure $\mu$ on $\Delta=B_{V M\left(\mathcal{H}_{\alpha}, \mathbb{R}\right)} \times B_{E^{*}}$ such that

$$
\psi(f \check{\otimes} e)=\int_{\Delta} p(f) e^{*}(e) d \mu\left(p, e^{*}\right)
$$

for each $f \check{\otimes} e \in \beta_{\alpha}^{\circ}(X, E)$ (see [8, page 231, Theorem 5].)

We say that a Banach space $E$ has the Banach-Stone property if for any compact Hausdorff spaces $\Omega_{1}$ and $\Omega_{2}$, the space $C\left(\Omega_{1}, E\right)$ is linearly isometric with $C\left(\Omega_{2}, E\right)$ if and only if $\Omega_{1}$ and $\Omega_{2}$ are topologically homeomorphic. Jerison [14] proved that if $E$ is a real strictly convex Banach space, then $E$ has the strong Banach-Stone property (and hence the Banach-Stone property). Behrends [2] proved that every Banach space $E$, such that $Z(E)$ (the centralizer of $E$ ) is one dimensional, has the strong Banach-Stone property ([2, Theorem 8.11]). For example, if $E$ is nonzero and fulfills each of the conditions 
(i) $E$ is smooth

(ii) $E$ is strictly convex

(iii) $E$ has no nontrivial $M$-ideal

(iv) $E$ is reflexive and all $M$-summands of $E$ are trivial,

then $Z(E)$ is one dimensional ([2, Proposition 5.1]).

More recently Behrends and Pelant [3] proved that if $K$ is a strongly rigid compact Hausdorff space, then the Banach space $C(K)$ has the Banach-Stone property. Now, we prove the following theorem.

Theorem 3.2. Let $X$ and $Y$ be two perfectly normal spaces and $E$ be a Banach space with the Banach-Stone property. If $\beta_{\alpha}^{\circ}(X, E)$ and $\beta_{\alpha}^{\circ}(Y, E)$ are isometric, then the compact spaces $b_{\alpha} X$ and $b_{\alpha} Y$ are homeomorphic.

Proof. The Banach algebra $\beta_{\alpha}(X)$ is unitary and commutative. Thus there is a compact Hausdorff space $K$ such that $\beta_{\alpha}(X) \cong C(K)$. Moreover, we know that $K$ is the set of all multiplicative functionals on $\beta_{\alpha}(X)$. Suppose that $\mu$ is a member of $K$, therefore for each $f, g \in \beta_{\alpha}^{\circ}(X)$, we have

$$
\int_{X} f g d \mu=\int_{X} f d \mu \int_{X} g d \mu
$$

We show that $\mu=\delta_{x}$ for some suitable $x \in X$ or $\mu$ is zero on finite subsets of $X$. Suppose that these cases are not occurred. Thus, there exist $x$ and $y$ in $X$ such that $\mu(\{x\})=r \neq 0$ and $\mu(\{y\})=s \neq 0$. If $f=m \delta_{x} \neq 0$ and $g=n \delta_{y} \neq 0$, then by (2), we have $0=m r \cdot n s$ which is a contradiction. If we identify each $x \in X$ by $\delta_{x}$, then one can embed $X$ as a subspace of $K$. The relative topology on $X$ is induced by the weak ${ }^{*}$-topology, the topology which is induced by Baire- $\alpha$ functions and has as a subbase

$$
B_{f, n}=\left\{x \in X:|f(x)|<\frac{1}{n}\right\}, \quad f \in \beta_{\alpha}^{\circ}(X) .
$$

Note that these sets are all of $\mathcal{P}_{\alpha}$ sets in $X$. The set $\{x\}$ is a $\mathcal{P}_{\alpha}$ set for each $x \in X$, therefore this topology is discrete, and $K$ is exactly the compactification $b_{\alpha} X$ of $X$, as in [6]. Suppose that $\beta_{\alpha}^{\circ}(X, E)$ and $\beta_{\alpha}^{\circ}(Y, E)$ are linear isometric.

By Theorem 3.1, we have

$$
\beta_{\alpha}^{\circ}(X, E)=\beta_{\alpha}^{\circ}(X) \check{\otimes} E=C\left(b_{\alpha} X\right) \check{\otimes} E=C\left(b_{\alpha} X, E\right) .
$$

The last equality is obtained from ([8, page 231, Theorem 5]). Hence, we have $C\left(b_{\alpha} X, E\right) \cong C\left(b_{\alpha} Y, E\right)$. Since the Banach space $E$ has the Banach-Stone property, thus spaces $b_{\alpha} X$ and $b_{\alpha} Y$ are homeomorphic.

Acknowledgement. The authors are grateful to the referees for their helpful comments. The second author is partially supported by the Center of Excellence for Mathematics(University of Isfahan). 


\section{References}

[1] Alo, R. A. and H. L. Shapiro: Normal Topological Spaces. Camb. Univ. Press 1974.

[2] Behrends, E.: M-Structure and the Banach-Stone Theorem. Lecture Notes Math. 736. Berlin: Springer 1979.

[3] Behrends, E. and J. Pelant: The cancellation law for compact Hausdorff spaces and vector-valued Banach-Stone theorems. Arch. Math. 64 (1995), 341 - 343.

[4] Cambern, M.: A Holsztynski theorem for spaces of continuous vector-valued functions. Studia Math. 58 (1978), $213-217$.

[5] Cengiz, B.: A generalization of the Banach-Stone theorem. Proc. Amer. Math. Soc. $40(1973)(2), 426-430$.

[6] Choban, M. M.: Isomorphism problem for the Baire function spaces of topological spaces. Serdica Math. J. 24 (1998), 5 - 20.

[7] Dashiell, F. K.: Isomorphism problems for the Baire classes. Pacific J. Math. 52 (1974), $29-43$.

[8] Diestel, J. and J. J. Uhlr, Jr.: Vector Measures. Math. Surveys 15. Providence (R.I.): Amer. Math. Soc. 1977.

[9] Engelking, R.: General Topology. Warszawa: PWN-Polish Sci. Publ. 1977.

[10] Gillman, L. and M. Jerison: Rings of Continuous Functions. Princeton: D. Van Nostrand Co., Inc., 1960.

[11] Jarosz, K.: Into isomorphisms of continuous functions. Proc. Amer. Math. Soc. $90(1984)(3), 373-377$.

[12] Jayne, J. E.: Space of Baire functions 1. Ann. Inst. Fourier (Grenoble) 24 (1974)(4), $47-76$.

[13] Jayne, J. E.: The class a Baire functions. Bull. Amer. Math. Soc. 80 (1974), $1151-1156$.

[14] Jerison, M.: The space of bounded maps into a Banach space. Ann. Math. 52 (1950), $309-327$.

[15] Kuratowski, K.: Topology 1. New York: Acad. Press 1966.

[16] Lacruz, M. and J. G. Llavona: Composition operator between algebras of uniformly continuous functions. Arch. Math. 69 (1997), 52 - 56.

[17] Natanson, I. P.: Theory of Functions of a Real Variable II. New York: Ungar Publ. Co. 1960.

[18] Rolewicz, S.: On inversion of non-linear transformations. Studia Math. 17 (1958), $79-83$.

[19] Shatery, H. R. and J. Zafarani: The equality between Borel and Baire classes. Real Anal. Exchange 30 (2005)(1), 373 - 384.

Received 13. 8. 2004 